\title{
Ueber die Constitution der einfachsten Eiweissstoffe. $\left.{ }^{1}\right)$
}

\author{
Von \\ A. Kossel.
}

Aus meinen früheren Arbeiten über die Protamine haben sich neue Anschauungen über die Constitution der Eiweisskörper ergeben. Die Untersuchungen über die Eigenschaften und die Spaltungsprodukte dieser bisher wenig beachteten und in ihrem chemischen Bau unbekannten Basen haben mich zu der Ansicht geführt, dass wir in ihnen die einfachsten Eiweisskörper vor uns haben. Ein zur Protamingruppe gehöriger Atomcomplex liegt nach meiner Auffassung auch dem Molekül der complicirteren Eiweisskörper zu Grunde und die chemische Kenntniss der Protamine scheint mir daher der Schlüssel zur Kenntniss des Eiweissmoleküls zu sein.

Das erste Protamin wurde von Miescher in den Spermatozoen des Lachses aufgefunden und im Jahre 1874 bekannt gemacht. Ich habe dann vor Kurzem gezeigt, dass in den Spermatozoen des Störs eine ähnliche Substanz vorkommt, der ich den Namen Sturin gegeben habe, während ich das Lachsprotamin als Salmin bezeichne. In dem Sperma des Herings fand ich nun ein Material, welches ebenfalls ein Protamin liefert, das ich mit dem Namen Clupein bezeichnet habe.

1) Die Resultate dieser Untersuchungen wurden bereits in den Sitzungsberichten der Gesellschaft zur Beförderung der gesammten Naturwissenschaften in Marburg vom 7. Juli 1897 und vom 16. März 1898 mitgetheilt. 
Um eine Vorstellung von der chemischen Natur dieser Körpergruppe zu gewinnen, schien es mir nothwendig, die drei genannten Protamine gleichzeitig nebeneinander zu untersuchen. Es wurde daher auch das früher beschriebene Salmin nochmals dargestellt und analysirt.

\section{Darstellung der Protaminsulfate.}

Die reifen Testikel des Herings, des Lachses oder des Störs werden nach dem früher beschriebenen Verfahren ${ }^{1}$ ) zur Gewinnung der Spermatozoenmasse behandelt, der Essigsäureniederschlag mit Alkohol und Aether ausgezogen. Etwa 100 gr. der trockenen, mit Alkohol und Aether extrahirten Masse. werden sodann mit $500 \mathrm{ccm}$. einer einprocentigen Schwefelsäure eine Viertelstunde geschüttelt, filtrirt und die Extraction des Filterrückstandes 6 Mal wiederholt. Das abfiltrirte Extract wird mit der dreifachen Menge Alkohol gefällt, die Flüssigkeit decantirt und abgesaugt, der Niederschlag in heissem Wasser gelöst und die Alkoholfällung wiederholt. Löst man nun den Niederschlag des Protaminsulfats in etwa $1 \frac{1 / 2}{2}$ Liter heissen Wassers und lässt die Lösung erkalten, so scheidet sich ein kleiner Theil des Sulfats als gelb oder bräunlich gefärbtes Oel ab. Dieser am schwersten lösliche Theil des Protaminsulfats wird ausgeschieden, die über dem Oel befindliche Flüssigkeit abgetrennt, auf ein kleines Volumen eingedampft und zur Gewinnung der Hauptmenge des Oels im Scheidetrichter hingestellt. Diese mittlere Fraction des Oels wurde für die folgenden Versuche allein benutzt.

Sie wurde von den letzten Spuren von Nucleinsäure, die ihr hartnäckig anhaften, in folgender Weise befreit: Das in warmem Wasser gelöste Protaminsulfat wurde mit Natriumpikrat ausgefällt, der gut ausgewaschene Niederschlag bei Gegenwart eines Ueberschusses von Schwefelsäure durch Ausschütteln mit Aether von Pikrinsäure befreit und das Protaminsulfat aus der schwefelsauren Lösung mit Alkohol gefällt. Diese Alkoholfällung wurde gewöhnlich noch einmal wiederholt. Das Protaminsulfat soll als ein lockerer weisser Niederschlag

1) Diese Zeitschrift, Bd. 22, S. 176. 
fallen; hat die Fällung klebrige Beschaffenheit, so muss die Lösung in Wasser und die Ausfällung durch Alkohol wiederholt werden. Die Darstellung verläuft bei Salmin und Clupein völlig gleich, das Sturin ist in Wasser leichter löslich und man muss daher die Lösung weiter eindampfen, um die Abscheidung des Oels zu erzielen.

\section{Das Clupein.}

Es wurden drei Präparate des Clupeinsulfats dargestellt und für die Analyse bei 110-120 getrocknet.

Die Darstellung von Präparat I war insofern eine abweichende, als hier die Fällung mit Pikrinsäure nicht stattgefunden hatte.

Die Analysen der bei $110-120^{\circ}$ getrockneten Substanz ergaben folgende Resultate:

\section{Präparat I.}

1. $0,1998 \mathrm{gr}$. Substanz gaben $0,2768 \mathrm{gr} . \mathrm{CO}_{2}$ und $0,1214 \mathrm{gr} . \mathrm{H}_{2} \mathrm{O}$.

2. $0,2124 \mathrm{gr}$. Substanz gaben $44,7 \mathrm{ccm}$. $\mathrm{N}_{2}$ bei $10,5^{\circ}$ und $753 \mathrm{~mm}$. Bar.

3. $0,2181 \mathrm{gr}$. Substanz gaben $47,1 \mathrm{ccm} . \mathrm{N}_{2}$ bei $12^{\circ}$ und $746 \mathrm{~mm}$. Bar.

\section{Präparat II.}

1. 0,1834 gr. Substanz gaben 0,2527 gr. $\mathrm{CO}_{2}$ und $0,1152 \mathrm{gr} . \mathrm{H}_{2} \mathrm{O}$.

2. $0,1748 \mathrm{gr}$. Substanz gaben $37,9 \mathrm{ccm} . \mathrm{N}_{2}$ bei $14^{\circ}$ und $741 \mathrm{~mm}$. Bar.

3. 0,1848 gr. Substanz gaben $41,1 \mathrm{ccm} . \mathrm{N}_{2}$ bei $18^{\circ}$ und $746 \mathrm{~mm}$. Bar.

4. 0,2666 gr. Substanz gaben 0,1334 gr. $\mathrm{Ba}_{4}$.

5. 0,2773 gr. Substanz gaben 0,1413 gr. $\mathrm{BaSO}_{4}$.

Präparat III.

1. 0,1942 gr. Substanz gaben 0,2699 gr. $\mathrm{CO}_{2}$ und 0,1164 gr. $\mathrm{H}_{2} \mathrm{O}$.

2. 0,2086 gr. Substanz gaben 0,2904 gr. $\mathrm{CO}_{2}$ und $0,1250 \mathrm{gr} . \mathrm{H}_{2} \mathrm{O}$.

3. 0,5874 gr. Substanz gaben 0,2872 gr. $\mathrm{BaSO}_{4}$.

\begin{tabular}{|c|c|c|c|c|c|c|c|c|}
\hline \multirow[b]{2}{*}{ C } & \multicolumn{3}{|c|}{ Präparat I. } & \multicolumn{3}{|c|}{ Präparat II. } & \multicolumn{2}{|c|}{ Präparat III. } \\
\hline & 37,78 & & & 37,58 & & & 37,90 & 37,96 \\
\hline $\mathrm{H}$ & 6,74 & & & 6,98 & & & 6,66 & 6,66 \\
\hline $\mathrm{N}$ & & 24,91 & 25,15 & & 24,86 & 25,21 & & \\
\hline $\mathrm{H}_{2} \mathrm{SO}_{4}$ & & & & & 21,04 & 21,43 & & \\
\hline
\end{tabular}




\begin{tabular}{ccc}
\multicolumn{2}{c}{ Berechnet für } & Gefunden \\
$\mathrm{C}_{30} \mathrm{H}_{57} \mathrm{~N}_{17} \mathrm{O}_{6} 2 \mathrm{H}_{2} \mathrm{SO}_{4}$ & im Mittel : \\
$\mathrm{C}$ & 38,02 & 37,85 \\
$\mathrm{H}$ & 6,44 & 6,76 \\
$\mathrm{~N}$ & 25,13 & 25,03 \\
$\mathrm{H}_{2} \mathrm{SO}_{4}$ & 20,70 & 21,01
\end{tabular}

Demnach ist die Formel: $\mathrm{C}_{30} \mathrm{H}_{57} \mathrm{~N}_{17} \mathrm{O}_{6}$ als der einfachste Ausdruck für die Zusammensetzung des Clupeins anzusehen.

Das Clupeinsulfat ist in trockenem Zustand ein weisses Pulver, welches sich sehr leicht in warmem Wasser auflöst. Kühlt man diese Lösung auf Zimmertemperatur ab, so scheidet sich, wenn die Lösung nicht $\mathrm{zu}$ verdünnt ist, ein farbloses, stark lichtbrechendes Oel ab, welches dadurch entsteht, dass das Clupeinsulfat, während es sich aus der Lösung abscheidet, ungetähr das gleiche Gewicht an Wasser löst. Dieses Oel trocknet beim Stehen an der Luft, oder schneller beim Erwärmen, zu einer farblosen, leicht zerreiblichen Masse ein, indem es etwa die Hälfte seines Gewichts an Wasser verliert. Sowohl das $\mathrm{Oel}$, wie die wässerige Lösung des Clupeins drehen die Ebene des polarisirten Lichtes stark nach links (s. unten).

Eine Lösung des freien Clupeins wird als stark alkalisch reagirende Flüssigkeit erhalten, wenn man die Lösung seines Sulfats mit der äquivalenten Menge Barytwasser ausfällt.

Das Clupein gibt ebenso wie die bisher beschriebenen Protamine eine blau-violette Biuretreaction, aber keine Reaction mit Millon's Reagens. Löst man Clupein in Eisessig und erwärmt mit concentrirter Schwefelsäure, so tritt keine Rothfärbung ein.

Die Clupeinsalze werden ebenso wie die übrigen Protamine gefällt durch phosphorwolframsaures, wolframsaures, pikrinsaures, chromsaures, ferrocyanwasserstoffsaures Alkali. Alle diese Fällungsmittel bewirken schon in neutraler, ja selbst in schwach alkalischer Lösung einen Niederschlag, ein Verhalten, welches unter Umständen zur Unterscheidung von Propeptonen und Peptonen dienen kann. Jodjodkalium und 
Bromwasser rufen ebenfalls Fällungen hervor, durch letzteres Reagens wird ein Additionsprodukt gebildet, welches schon beim Umlösen wieder das ganze Brom verliert.

Auch Salze schwerer Metalle, z. B. Quecksilberchlorid, erzeugen Niederschläge. Versetzt man eine Lösung des freien Clupeins mit überschüssigem Silbernitrat, so bildet sich keine Fällung, fügt man tropfenweise Natronhydrat hinzu, so entsteht ein weisser Niederschlag eines Silbersalzes. Wenn die Menge des zugefïgten Natrons die Hälfte des Aequivalents vom Clupein übersteigt, so nimmt bei weiterem Zusatz von Natron der Niederschlag mehr und mehr eine gelbe Farbe an, indem sich dem Clupeinsilber Silberoxyd beimischt.

Kupferoxydul bildet mit Clupein eine schwer lösliche Verbindung, wie dies schon von Balke in Bezug auf das Salmin angegeben ist. Man erhält also einen Niederschlag, wenn man die Lösung des Clupeinsulfats mit Kupfersulfat und Natriumhyposulfit versetzt. Kupfersulfat allein ruft keine Fällung hervor.

Ebenso wie die übrigen Protamine gibt auch das Clupein in ammoniakalischer Lösung einen Niederschlag mit coagulirbaren Eiweisskörpern und mit Propeptonen ("primären Albumosen").

Das Clupein ist eine starke Base, deren wässerige Lösung, wie schon erwähnt, kräftig alkalische Reaction besitzt. Auch das Carbonat, das Chlorid und Nitrat des Clupeins lassen sich darstellen. Diese Salze sind in Wasser leicht löslich und aus dieser Lösung durch Alkohol nur unvollständig niederzuschlagen.

\section{Das Salmin.}

Um die Beziehungen des Salmins zum Clupein aufzuklären, erschien mir eine erneute Darstellung und Analyse des Salminsulfats wünschenswerth.

Diese Darstellung wurde in der oben beschriebenen Weise ausgeführt, die Analysen ergaben folgende Załien für die bei $120^{\circ}$ getrocknete Substanz:

1. 0,2205 gr. Substanz gaben 0,3012 gr. $\mathrm{CO}_{2}$ und 0,1296 gr. $\mathrm{H}_{2} \mathrm{O}$.

2. 0,2177 gr. Substanz gaben 0,2973 gr. $\mathrm{CO}_{2}$ und 0,1276 gr. $\mathrm{H}_{2} \mathrm{O}$. 
3. $0,1661 \mathrm{gr}$. Substanz gaben $35,3 \mathrm{ccm}$. Stickstoff bei $14,0^{\circ}$ und $757 \mathrm{Bar}$.

4. 0,4256 gr. Substanz gaben 0,2093 gr. $\mathrm{BaSO}_{4}$.

\begin{tabular}{c|r|r|r|l}
\hline \hline $\mathrm{C}$ & 37,25 & 37,24 & & \\
\hline $\mathrm{H}$ & 6,53 & 6,51 & & \\
\hline $\mathrm{N}$ & & & & 24,90 \\
\hline $\mathrm{H}_{2} \mathrm{SO}_{4}$ & & & 20,67 &
\end{tabular}

$\begin{array}{rrr} & \text { Gefunden } \\ & \mathrm{C} & 37,25 \\ \mathrm{H} & 6,52 \\ \mathrm{~N} & 24,90 \\ \mathrm{H}_{2} \mathrm{SO}_{4} & 20,67\end{array}$

Berechnet füir

$\mathrm{C}_{30} \mathrm{H}_{59} \mathrm{~N}_{17} \mathrm{O}_{7}+2 \mathrm{H}_{2} \mathrm{SO}_{4}$

37,31

6,54

24,66

20,31

Hiernach unterscheidet sich das Salminsulfat vom Clupeinsulfat nur durch den Mehrgehalt eines Moleküls Wasser.

Miescher, Piccard, Schmiedeberg und ich haben früher andere Formeln für das Salmin aufgestellt. Die obige Formel $\mathrm{C}_{30} \mathrm{H}_{59} \mathrm{~N}_{17} \mathrm{O}_{6}, 2 \mathrm{H}_{2} \mathrm{SO}_{4}$ lässt sich aber auch mit den früheren Analysenwerthen in Einklang bringen, wenn man die folgenden Erwägungen macht.

Erstens existiren die Protamine, wie viele ähnliche Stoffe, in mehreren Hydrationsstufen, die leicht in einander übergehen. Man wird also bei Analysen desselben Präparats verschiedene Werthe erhalten, welche sich um $\mathrm{H}_{2} \mathrm{O}$ unterscheiden, je nachdem man mehr oder minder scharf getrocknet hat.

Zweitens kommt es bei diesen Stoffen leicht zur Bildung basischer Salze; dies scheint bei dem von mir früher dargestellten und von Herrn Wolkowicz im Winter 1894/95 analysirten Sulfat der Fall gewesen zu sein. In Folge dessen ist der Schwefelsäuregehalt dieses Präparats etwas zu niedrig. Aehnliches ist wohl auch bei Miescher's Salminplatinchlorid anzunehmen.

Am klarsten erscheinen die Verhältnisse, wenn man die von mir für das Clupein aufgestellte Formel $\mathrm{C}_{30} \mathrm{H}_{57} \mathrm{~N}_{17} \mathrm{O}_{6}$ der Betrachtung zu Grunde legt. Dann ist die Formel des Salminsulfats nach meinen letzten Analysen folgendermassen $\mathrm{zu}$ schreiben: $\mathrm{C}_{30} \mathrm{H}_{57} \mathrm{~N}_{17} \mathrm{O}_{6}, 2 \mathrm{H}_{2} \mathrm{SO}_{4}+\mathrm{H}_{2} \mathrm{O}$. 
Das Salminsulfat, dessen Analyse ich im Jahre 1897 mitgetheilt habe, scheint in Folge schärferen Trocknens ärmer an Wasser gewesen $\mathrm{zu}$ sein und entspricht annähernd der Formel

$$
\mathrm{C}_{30} \mathrm{H}_{57} \mathrm{~N}_{17} \mathrm{O}_{6}, 2 \mathrm{H}_{2} \mathrm{SO}_{4}-\mathrm{H}_{2} \mathrm{O} \text {, }
$$

wie aus folgender Zusammenstellung hervorgeht.

$$
\begin{array}{ccccc}
\text { Berechnet für } & \mathrm{C} & \mathrm{H} & \mathrm{N} & \mathrm{H}_{2} \mathrm{SO}_{4} \\
\mathrm{C}_{30} \mathrm{H}_{55} \mathrm{~N}_{17} \mathrm{O}_{5}, 2 \mathrm{H}_{2} \mathrm{SO}_{4}: & 38,75 & 6,35 & 25,62 & 21,10 \\
\text { Gefunden (Mittel)1): } & 38,45 & 6,95 & 25,20 & 19,592)
\end{array}
$$

\begin{tabular}{|c|c|c|c|}
\hline $\begin{array}{r}\text { Ber } \\
\mathrm{C}_{30} \mathrm{H}_{57} \mathrm{~N}_{17} \mathrm{O}_{6}\end{array}$ & $\begin{array}{l}\text { anet für } \\
\mathrm{HCl},+2 \mathrm{PtCl}_{4} \text {. }\end{array}$ & & $\begin{array}{l}\text { en } \\
\text { d) }\end{array}$ \\
\hline $\mathrm{C}$ & 22,97 & 22,80 & 22,78 \\
\hline $\mathrm{H}$ & 3,89 & 4,15 & 4,40 \\
\hline $\mathrm{N}$ & 15,16 & & \\
\hline $\mathrm{Pt}$ & 24,80 & & \\
\hline CI & 27,2 & & \\
\hline
\end{tabular}

Am besten stimmen die Analysen Piccard's ${ }^{3}$ ) vom Platindoppelsalz mit dieser Formel überein.

Auch diejenigen Zahlen, welche Cloetta in Miescher's hinterlassenen Präparaten fand, ${ }^{4}$ ) passen zu meiner Formel.

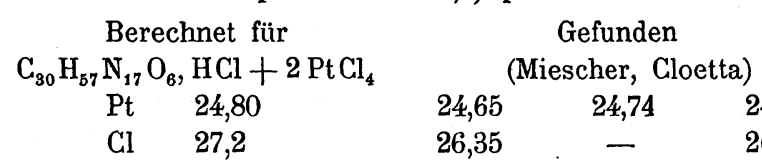

$\begin{array}{ccc}24,65 & 24,74 & 24,36 \\ 26,35 & - & 26,97\end{array}$

Ausserdem liegen noch die Analysen zweier Platinsalze von Miescher vor. Die ersten stammen aus dem Jahr 1874 ${ }^{5}$ ) und haben für das Platin Werthe ergeben, welche zwischen $23,13 \% \mathrm{Pt}$ und 24,64\% Pt schwanken. Andere Analysen des von Mies cher dargestellten Platinsalzes sind erst 1896 publicirt worden. ${ }^{6}$ ) Zieht man von den älteren Analysen Miescher's diejenigen in Betracht, welche die höheren, mit Piccard's und

1) Diese Zeitschrift, Bd. 22 S. 179.

2) s. vorige Seite.

3) Berichte der deutschen chemischen Gesellschaft, Bd. VII, S. 17, 18.

4) Archiv für experiment. Pathologie u. Pharmakologie, Bd. 37, Miescher, Histochemische und physiologische Arbeiten, II S. 366.

5) Verhandl. d. naturforsch. Gesellschaft in Basel, Bd. VI S. 138 bis 208, 1874.

6) Archiv für experiment. Pathol. und Pharmakol., Bd. 37, S. 100. 
Cloetta's Analysen übereinstimmenden Werthe für Platin ergaben, so kommt man zu einer Formel, welche ein Molekül Wasser weniger enthält wie die oben angeführte.

\begin{tabular}{|c|c|c|c|c|c|}
\hline Berechnet für & G & $\mathrm{H}$ & $\mathrm{N}$ & $\mathrm{Pt}$ & $\mathrm{Cl}$ \\
\hline $\mathrm{C}_{30} \mathrm{H}_{55} \mathrm{~N}_{17} \mathrm{O}_{5}, 4 \mathrm{HCl}+2 \mathrm{PtCl}_{4}$ & 23,2 & 3,81 & 15,3 & 25,09 & 27,4 \\
\hline 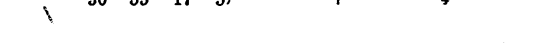 & $(23,16$ & 4,35 & $15,001)$ & 24,64 & \\
\hline fefunden I. Aeltere Analysen Miescher's: & $\{23,21$ & 4,29 & & 24,53 & \\
\hline & 23,11 & 4,25 & & & \\
\hline II. Neuere & 23,40 & 4,26 & 15,11 & 24,07 & 26,68 \\
\hline
\end{tabular}

Aus dieser Zusammenstellung ergibt sich, dass sich die von mir aufgestellte Formel auch im Einklang mit den Resultaten anderer Forscher befindet.

Es ist für die weiteren Betrachtungen gleichgiltig, ob man die Formel des Salmins $\mathrm{C}_{30} \mathrm{H}_{57} \mathrm{~N}_{17} \mathrm{O}_{6}$ oder $\mathrm{C}_{30} \mathrm{H}_{55} \mathrm{~N}_{17} \mathrm{O}_{5}$ schreibt; je nachdem man mehr oder weniger trocknet, wird man Präparate der einen oder anderen Zusammensetzung erhalten.

Um die Frage nach der Identität von Salmin und Clupein zu entscheiden, untersuchte ich weiterhin einige Eigenschaften der Sulfate. Zunächst stellte ich die Löslichkeit in Wasser bei gewöhnlicher Temperatur fest, indem ich in der gesättigten Lösung der Sulfate den Stickstoffgehalt mit der Kjeldahl'schen Methode bestimmte. Hierbei ergab sich folgendes Resultat:

Clupeinsulfat Salminsulfat

Stickstoff in $100 \mathrm{ccm}$. der gesättigten Lösung: $0,3248 \quad 0,3206$

$$
\left.\begin{array}{c}
\text { Daraius berechneter Gehalt an } \\
\mathrm{C}_{30} \mathrm{H}_{57} \mathrm{~N}_{17} \mathrm{O}_{6}, 2 \mathrm{H}_{2} \mathrm{SO}_{4}
\end{array}\right\} 1,29 \% \quad 1,27 \%
$$

Demnach stimmt das Salmin mit dem Clupein hinsichtlich seiner Löslichkeit bei gewöhnlicher Temperatur überein.

Auch die Einwirkung beider Lösungen auf das polarisirte Licht war die gleiche. Bei der Untersuchung der gesättigten Lösungen im $60 \mathrm{~cm}$. langen Rohr ergaben sich folgende Drehungswinkel :

$$
\begin{array}{cc}
\text { Clupeinsulfat } & \text { Salminsulfat } \\
-6,43^{\circ} & -6,17^{\circ}
\end{array}
$$

1) Verbrennung mit Natronkalk. 
Der Brechungscoefficient des aus der Lösung abgeschiedenen wasserhaltigen Oels war folgender:

$\begin{array}{cc}\text { Clupeinsulfat } & \text { Salminsulfat } \\ 1,4430 & 1,4420\end{array}$

Diese Untersuchungen führen zu dem Resultat, dass Salmin und Clupein identisch sind.

Hering und Lachs erzeugen also in ihren Testikeln das gleiche Protamin. Nach den Untersuchungen, welche Herr A. Mathews im hiesigen Laboratorium ausgeführt hat, sind die chemischen Verhältnisse der Spermatozoen bei beiden Fischen überhaupt die gleichen ${ }^{1}$ ).

\section{Das Sturin.}

Wenn man nach der oben beschriebenen Methode die Testikel des Störs verarbeitet, so erhält man ein Protamin, welches sich vom Salmin und Clupein durch grössere Löslichkeit unterscheidet, im Uebrigen aber ein ähnliches Verhalten zeigt. Da die Störhoden eine noch unbekannte Substanz enthalten, welche sich leicht unter Bildung eines rothen Farbstoffs zerlegt, so ist das als Oel abgeschiedene Sulfat zunächst stark gefärbt. Dieser Farbstoff lässt sich aber durch Kochen der wässerigen Lösung mit Thierkohle entfernen.

Die Sturinsalze können ebenso wie die Salze des Salmins (resp. des Clupeins) aus ihren Lösungen ausgesalzen werden. Das Sturinchlorid wird z. B. durch Kochsalz aus seinen Lösungen ausgefällt, doch ist hierzu eine grössere Concentration an $\mathrm{NaCl}$ nöthig, als zur Ausfällung des Salminchlorids. Sehr auffallend ist das Verhalten des gelösten Sturinsulfats zu Aether. Fügt man zu einer Lösung von Sturinsulfat eine geringe Menge Aether oder einige Tropfen Alkohol oder Aceton, so erfolgt sofort die Ausscheidung des Sulfats als ölige Masse, die sich allmählich am Boden des Gefässes ansammelt.

Das Sturinsulfat gab bei der Analyse folgende Zahlen:

1. 0,2277 gr. Substanz gaben 0,3128 gr. $\mathrm{CO}_{2}$ und 0,1332 gr. $\mathrm{H}_{2} \mathrm{O}$.

2. 0,2358 gr. Substanz gaben 0,3232 gr. $\mathrm{CO}_{2}$ und 0,1384 gr. $\mathrm{H}_{2} \mathrm{O}$.

3. $0,1821 \mathrm{gr}$. Substanz gaben $34,8 \mathrm{ccm} . \mathrm{N}_{2}$ bei $13^{\circ}$ und $761 \mathrm{~mm}$. Bar.

4. $0,1828 \mathrm{gr}$. Substanz gaben $35,0 \mathrm{ccm} . \mathrm{N}_{2}$ bei $12,5^{\circ}$ und $760 \mathrm{~mm}$. Bar.

5. 0,2946 gr. Substanz gaben 0,1590 gr. $\mathrm{BaSO}_{4}$.

1) Diese Zeitschrift Bd. 23, S. 399. 


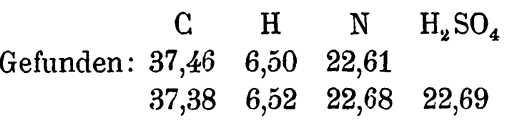

Berechnet

für $2 \mathrm{C}_{33} \mathrm{H}_{61} \mathrm{~N}_{17} \mathrm{O}_{7}+5 \mathrm{H}_{2} \mathrm{SO}_{4} \quad 37,64 \quad 6,37 \quad 22,62 \quad 23,29$

für $4 \mathrm{C}_{38} \mathrm{H}_{68} \mathrm{~N}_{19} \mathrm{O}_{7}+11 \mathrm{H}_{2} \mathrm{SO}_{4} \quad 37,61 \quad 6,49 \quad 23,16 \quad 23,46$.

Die zweite Formel muss mit Rücksicht auf die Resultate der weiter unten beschriebenen Spaltungsversuche als die wahrscheinlichere angesehen werden.

\section{Die nächsten Spaltungsprodukte der Protamine.}

Erhitzt man die Protamine mit verdünnter Schwefelsäure zum Sieden, so entstehen zuerst Produkte, welche ihnen hinsichtlich ihrer Eigenschaften noch nahestehen und welche man als Peptone der Protamine - «Protone» - zu betrachten hat; aus diesen gehen dann durch weitere Zersetzung die Basen hervor, welche ich schon früher als Zersetzungsprodukte des Sturins aufgefunden habe, nämlich Arginin und Histidin.

Neuerdings habe ich unter den Zersetzungsprodukten des Sturins noch einen Körper von der Zusammensetzung des Lysins nachweisen können.

Die Untersuehung der nicht krystallisirten Zwischenprodukte zwischen den Protaminen und den drei genannten Endprodukten ist noch nicht beendet, doch ergibt sich aus meinen bisherigen Untersuchungen, die ich später ausführlicher mitzutheilen gedenke, folgendes:

Kocht man Clupein eine Stunde lang mit Schwefelsäure (10 ccm. concentrirte Schwefelsäure mit $90 \mathrm{ccm}$. Wasser) am Rückflusskühler, so sind in der Lösung drei Körper nachzuweisen, die dem Clupein ähnlich sind, sich jedoch von diesem dadurch unterscheiden, dass ihre Sulfate in Wasser leicht löslich sind. Untersucht man diese Stoffe mit Hülfe der Fällungsreactionen der Protamine, so findet man, dass diese entweder überhaupt nicht eintreten oder dass die Niederschläge leichter löslich sind. Mit coagulirbarem Eiweiss und Propeptonen gaben diese Körper in ammoniakalischer Lösung keine Fällung, sondern höchstens eine geringe Trübung, die vielleicht von Spuren unzersetzten Protamins herrührt. 
Die Analysen dieser aus dem Clupein dargestellten Produkte, die noch nicht beendet sind, haben bisher ergeben, dass eines derselben die gleiche Zusammensetzung hat wie das Clupein, die anderen aber mehr Wasserstoff und weniger Kohlenstoff und Stickstoff besitzen. Man wird sie also als Hydrate des Clupeins betrachten müssen.

Eines derselben entspricht etwa der Formel $\mathrm{C}_{30} \mathrm{H}_{61} \mathrm{~N}_{17} \mathrm{O}_{8}$ und man muss hier den Eintritt von 2 Molekülen $\mathrm{H}_{2} \mathrm{O}$ in das Molekül des Clupeins annehmen. Merkwürdiger Weise fand sich die gleiche Zusammensetzung bei dem analogen Produkt aus Sturin.

Gegenüber diesen Hydrationsvorgängen erschien es mir interessant, zu untersuchen, ob aus den Protaminen noch wasserärmere Produkte dargestellt werden können. Es zeigte sich, dass bei längerem Erhitzen von freiem Sturin auf $150^{\circ}$ im Trockenschrank allerdings eine beträchtliche Gewichtsabnahme erfolgt, die aber nicht allein auf Verlust von Wasser, sondern auch auf Entweichen stickstoffhaltiger Substanzen zurückzuführen ist.

\section{Die Hexonbasen.}

Mit dem Namen «Hexonbasen» bezeichne ich die Gruppe derjenigen Basen, welche sich bei der Hydrolyse der Protamine bilden: Histidin, Arginin und die Lysine, während ich unter der Bezeichnung "Hexone» die bei der Zersetzung der Eiweisskörper entstehenden stickstoffhaltigen Verbindungen mit 6 Atomen Kohlenstoff, also ausser den Hexonbasen auch das Leucin, zusammenfasse. ${ }^{1}$ )

Diese Bezeichnung soll einer bedeutungsvollen Uebereinstimmung in dem Bau stickstoffhaltiger und stickstofffreier organischer Bestandtheile der lebenden Wesen Ausdruck verleihen. Ebenso wie die Gliederung in dem Molekül der meisten Kohlenhydrate eine solche ist, dass Gruppen von sechs

1) Die Endung aon» ist seit langer Zeit für Eiweissderivate (Propepton, Pepton) gebräuchlich, und ich glaube sie daher auch für diese Spaltungsprodukte der Eiweisskörper benutzen zu dürfen, trotzdem nach der Nomenclatur einiger Chemiker die Bezeichnung \&Hexon» auf einen Kohlenwasserstoff $\mathrm{C}_{6} \mathrm{H}_{8}$ angewendet werden sollte. 
Kohlenstoffatomen eine Einheit bilden, die entweder selbstständig erscheinen oder doch beim Aufbau und bei dem Zerfall der complicirteren Stoffe als Durchgangsprodukte auftreten, so bilden die Körper mit sechs Atomen Kohlenstoff auch die wesentlichen Glieder des Eiweissmoleküls. Wir kennen derartige Zerfallsprodukte der Eiweissstoffe mit 1, 2, 3, 4 Atomen Stickstoff, deren basische Eigenschaften begreiflicher $\mathrm{W}^{\top}$ eise entsprechend der Zahl der Stickstoffatome zunehmen.

Diese Verbindungen sind folgende:

$$
\begin{array}{ll}
\text { Leucin } & \mathrm{C}_{6} \mathrm{H}_{13} \mathrm{NO}_{2} \\
\text { Lysin } & \mathrm{C}_{6} \mathrm{H}_{14} \mathrm{~N}_{2} \mathrm{O}_{2} \\
\text { Histidin } & \mathrm{C}_{6} \mathrm{H}_{9} \mathrm{~N}_{3} \mathrm{O}_{2} \\
\text { Arginin } & \mathrm{C}_{6} \mathrm{H}_{14} \mathrm{~N}_{4} \mathrm{O}_{2}
\end{array}
$$

Aus den Protaminen entstehen nur die drei leizteren Körper.

Das Verfahren, nach welchem ich diese Basen aus den Protaminen dargestellt habe, beruht auf folgenden Fällungsreactionen.

1. Trennung des Histidins vom Arginin und Lysin.

Kohlensaures Histidin wird bei Abwesenheit von neutralen Alkalisalzen selbst in sehr verdünnten Lösungen durch Quecksilberchlorid gefällt, kohlensaures Arginin und Lysin werden hingegen aus hinreichend verdünnten Lösungen nicht niedergeschlagen. Versetzt man eine verdünnte Lösung des freien Arginins mit Quecksilberchlorid, so erhält man einen Niederschlag, der sich beim Durchleiten von Kohlensäure allmählich auflöst; der in einer Histidinlösung erzeugte Niederschlag löst sich hingegen nicht.

Um zu untersuchen, inwiefern diese Fällung auch zur quantitativen Trennung von Histidin und Arginin benutzt werden kann, stellte ich folgenden Versuch an.

Ich benutzte ein Präparat von basisch kohlensaurem Arginin, in welchem durch eine Kjeldahl-Bestimmung die Menge des Stickstoffs festgestellt war. Von diesem Präparat wurde eine Menge, die 0,2860 gr. Arginin entsprach, abgewogen und mit 0,1020 gr. Histidin in Wasser gelöst. Die Lösung wurde 
mit Kohlensäure gesättigt, dann mit einer wässerigen Lösung von Quecksilberchlorid ausgefällt und im Niederschlag die Menge des Stickstoffs nach Kjeldahl unter Innehalten der durch Gegenwart des Quecksilbers bedingten Cautelen bestimmt. Dieselbe ergab 0,0204 gr. Stickstoff, d. i. 0,090t gr. Histidin. Es war also ein Verlust von 0,0116 gr. Histidin durch die Löslichkeit der Quecksilberverbindung eingetreten. Dieser Verlust ist ein so geringer, dass man die Fällung zur annähernd quantitativen Bestimmung benutzen kann. Nur wenn die Fällung aus einer Lösung von Arginincarbonat erzeugt ist, welche mehr als 1 Theil Arginin auf 500 Theile Wasser erhält, ist eine erhebliche Beimengung von Arginin zum Histidin zu befürchten.

2. Trennung von Arginin und Lysin.

Das Verfahren beruht darauf, dass bei der Einwirkung von Silbersalzen auf Arginin bei gewöhnlicher Temperatur eine silberhaltige Base entsteht, welche durch fixes Alkali als solche gefällt wird. Dasselbe ist übrigens auch beim Histidin der Fall. Lässt man zu einer Lösung von Argininnitrat aus einer Bürette allmählich Silbernitrat hinzufliessen, so tritt kein Niederschlag ein, solange die Flüssigkeit sauer ist. Entnimmt man nun mit einem Glasstab einen Tropfen der Lösung und lässt ihn in ein mit reiner Natronlauge gefülltes Uhrgläschen vom Rande her hineinfliessen, so beobachtet man je nach der Menge des hinzugefügten Silbernitrats verschiedene Erscheinungen. Ist die Menge des hinzugefügten Silbernitrats gering, so entsteht im Uhrgläschen gar kein Niederschlag, nach weiterem Zusatz von Silbernitrat bemerkt man im Uhrgläschen einen weissen Niederschlag, der sich bald wieder löst; ist noch mehr Silber in der Flüssigkeit vorhanden, so bleibt die weisse Fällung bestehen, ist endlich ein Ueberschuss von Silber da, so entsteht eine gelbe silberoxydhaltige Fällung, die sich bald braun färbt.

Macht man denselben Versuch mit Lysin, so bemerkt man entweder überhaupt keine Niederschlagbildung oder eine solche, welche sich leicht im Ueberschuss von Natron löst, oder nachdem mehr Silber hinzugefügt worden ist, eine Fällung 
von Silberoxyd. Es entsteht aber keine in Natron unlösliche weisse Silberverbindung des Lysins.

Sehr zweckmässig ist es, das Natron durch Baryt zu ersetzen. Dies hat den Vortheil, dass das Reagens aus dem Filtrat leicht $\mathrm{zu}$ entfernen ist und dass die Silberniederschläge besser filtriren.

Diese Methode bietet also die Möglichkeit einer Trennung von Arginin und Histidin einerseits und Lysin andererseits, und die Darstellung gestaltet sich sehr einfach, wenn man bei Anweindung des Baryts das Silbernitrat durch Silbersulfat ersetzt.

Zur Prüfung dieser Methode stellte ich zwei Versuche an.

1. Eine Lösung, welche 0,2710 gr. Arginin enthielt, wurde mit Silbersulfat und Natronlauge gefällt, im Niederschlag nach Kjeldahl eine Stickstoff-Bestimmung ausgeführt. Diese ergab 0,0822 gr. N, d. i. 0,2554 gr. Arginin, also einen Verlust von $0,0156 \mathrm{gr}$.

2. Eine 0,1701 gr. Arginin enthaltende Menge des basischen Carbonats wurde mit der aus 0,1 gr. Lysinchlorhydrat dargestellten Lösung von Lysin vermischt, mit Silbersulfat und Baryt ausgefällt und im Niederschlag nach Entfernung des Silbers mit $\mathrm{H}_{2} \mathrm{~S}$ eine Stickstoffbestimmung nach $\mathrm{Kjeldahl}$ ausgeführt. Diese ergab 0,04592 gr. N, d. i. 0,1427 gr. Arginin, entsprechend einem Verlust von 0,0274 gr. Arginin. Man wird also diese Fällung zur annähernd quantitativen Trennung des Arginins vom Lysin verwerthen können.

Mit Hülfe dieser Methoden konnte ich aus dem Salmin, Clupein und Sturin die Protaminbasen leicht in krystallisirtem Zustand darstellen. Das Verfahren wird folgendermassen ausgeführt.

Man kocht $20 \mathrm{gr}$. Protaminsulfat mit einer Mischung von $60 \mathrm{ccm}$. concentrirter Schwefelsäure und $120 \mathrm{ccm}$. Wasser 8 Stunden am Rückflusskühler. Hierdurch tritt keine Braunfärbung ein, wenn das Präparat hinreichend rein ist. Während die ursprüngliche Lösung die Ebene des polarisirten Lichts nach links ablenkt, zeigt sich nach der Vollendung dieser Reaction starke Rechtsdrehung. Man übersättigt die Flüssigkeit mit Barytwasser, leitet zur Entfernung des überschüssigen Aetzbaryts 
und zur Ueberführung der Basen in die Carbonate Kohlensäure hindurch und filtrirt den Niederschlag ab. Der Niederschlag wird sodann mehrmals mit Wasser ausgekocht, das Waschwasser dem Filtrat zugefügt.

Man fügt zu der alkalisch reagirenden Flüssigkeit jetzt eine concentrirte wässerige Lösung von Quecksilberchlorid hinzu, bis blaues Lakmuspapier durch dieselbe roth gefärbt wird. Der Niederschlag wird nach 24stündigem Stehen abgesaugt, mit Wasser ausgewaschen, sodann mit Schwefelwasserstoff zerlegt und die vom Schwefelquecksilber abfiltrirte Flüssigkeit zur Krystallisation des salzsauren Histidins eingedampft.

Das Filtrat des Quecksilberchlorid-Niederschlages wird zur Entfernung des Quecksilbers mit Schwefelwasserstoff behandelt. In die filtrirte, sodann zur Entfernung des Schwefelwasserstoffs eingedampfte und auf dem Wasserbade erwärmte Flüssigkeit, welche mindestens $1^{1 / 2}$ Liter beträgt, bringt man in kleinen Portionen Silbersulfat, bis das Chlor aus der Lösung entfernt ist, und prüft das Sulfat in folgender Weise: Man entnimmt demselben mit dem Glasstab einen Tropfen und lässt. diesen in ein mit reiner Natronlauge gefülltes Uhrgläschen hineinfliessen. Entsteht kein Niederschlag oder eine weisse Fällung, so ist die Menge des gelösten Silbersulfats noch nicht ausreichend. Man setzt sodann entweder in kleinen Portionen mehr Silbersulfat oder - falls dieses noch in ungelöstem $\mathrm{Zu}$ stand vorhanden - mehr Wasser hinzu und wiederholt die Prüfung. Sobald diese Probe eine gelbe Fällung mit Natronlauge ergibt, filtrirt man schnell die heisse Flüssigkeit vom Chlorsilber und dem etwa ungelöst vorhandenen Silbersulfat $a b$ und bringt in das abgekühlte Filtrat einen Ueberschuss von gepulvertem Aetzbaryt. Dieser ruft die Bildung eines hellbraunen Niederschlages hervor. Um festzustellen, ob die Menge des zugefügten Baryts ausreicht, filtrirt man eine Probe der Flüssigkeit $a b$ und versetzt das Filtrat mit einem grossen Ueberschuss von Barytwasser. Entsteht jetzt eine Fällung, so muss zu der Hauptmenge noch mehr Baryt hinzugefügt werden. Der gesammte durch Baryt erzeugte Silberniederschlag wird 
abgesaugt, vom Filter genommen, mit Wasser angerührt, sorgfältig ausgewaschen, sodann in Wasser vertheilt und mit Schwefelwasserstoff zerlegt, aus der eingedampften Flüssigkeit krystallisirt das freie Arginin, welches entsprechend seiner stark basischen Natur etwas Kohlensäure aus der Luft angezogen hat.

Das Filtrat wird mit Schwefelsäure angesäuert, durch Schwefelwasserstoff vom Silber befreit, die vom Baryumsulfat und Silbersulfid abfiltrirte Lösung durch Baryt genau ausgefällt und zur Krystallisation des Lysins eingedampft.

Die Analysen des aus Sturin dargestellten Histidins und. Arginins habe ich schon früher mitgetheilt, $\left.{ }^{1}\right)$ dieselben Basen wurden aus Salmin (resp. Clupein) gewonnen.

Aus dem Sturin stellte ich ein Salz von der Zusammensetzung des Lysinpikrats dar, indem ich die vom Argininsilber abfiltrirte Flüssigkeit zunächst durch Schwefelwasserstoff vom Silber befreite und sodann bei neutraler Reaction mit Natriumpikrat versetzte. Ich erhielt ein in Wasser schwer lösliches Pikrat, das nach dem Umkrystallisiren folgende Analysenwerthe der bei $120^{\circ}$ getrockneten Substanz gab:

1. 0,1691 gr. Substanz gab 0,2378 gr. $\mathrm{CO}_{2}$ und 0,0785 gr. $\mathrm{H}_{2} 0$.

2. 0,1825 gr. Substanz gab 0,2558 gr. $\mathrm{CO}_{2}$ und 0,0791 gr. $\mathrm{H}_{2} \mathrm{O}$.

3. 0,1829 gr. Substanz gab $29,8 \mathrm{ccm}$. Stickstoff bei $12^{0}$ und $738 \mathrm{~mm}$. Bar.

$\begin{array}{rccc} & \mathrm{C} & \mathrm{H} & \mathrm{N} \\ \text { Gefunden: } & 38,35 & 5,15 & 18,77 \\ & 38,22 & 4,81 & - \\ \text { Berechnet für } \mathrm{C}_{6} \mathrm{H}_{14} \mathrm{~N}_{2} \mathrm{O}_{2}, \mathrm{C}_{6} \mathrm{H}_{3} \mathrm{~N}_{3} \mathrm{O}_{7}: & 38,40 & 4,53 & 18,67\end{array}$

Aus diesem Pikrat wurde die Pikrinsäure durch Aether bei Gegenwart überschüssiger Salzsäure entfernt und die salzsaure Lösung zur Darstellung eines Platindoppelsalzes benutzt.

Die im Vacuum über Schwefelsäure getrocknete Substanz ergab bei der Analyse:

$0,1820 \mathrm{gr}$. Substanz gab 7,9 ccm. Stickstoff bei $10,5^{0}$ und $747 \mathrm{~mm}$. Bar.

Gefunden : $\tilde{5}, 1$

Berechnet für $\mathrm{C}_{6} \mathrm{H}_{14} \mathrm{~N}_{2} \mathrm{O}_{2}, \mathrm{H}_{2} \mathrm{PtCl}_{6}: \check{5}, 05$

1) Diese Zeitschrift, Bd. XXII, S. 176. 
Es ist somit hier ein Körper von der Zusammensetzung des Lysins vorhanden.

Auch aus Clupein konnte ich ein Pikrat darstellen, welches in seinen Eigenschaften vollkommen mit dem Pikrat aus Sturin übereinstimmte, doch war die Menge eine viel geringere, und deshalb ist der Körper aus Clupein bisher noch nicht analysirt worden.

Wegen der Schwierigkeit, das Chlorhydrat oder das Platindoppelsalz bei den geringen Substanzen analysenrein darzustellen, habe ich mich beim Clupein bisher mit folgendem Versuch begnügen müssen. Aus der vom Histidin und Arginin befreiten Flüssigkeit fällte ich die Base durch Phosphorwolframsäure heraus und krystallisirte den Niederschlag aus heissem Wasser um. In dieser Krystallmasse, die vermuthlich auch freie Phosphorwolframsäure enthielt, bestimmte ich Kohlenstoff und Stickstoff durch Elementaranalyse. Die gefundenen Mengen entsprechen dem Verhältniss $3 \mathrm{C}: \mathrm{N}$. Hiernach glaube ich annehmen zu dürfen, dass auch aus dem Clupein ein Körper von der Zusammensetzung des Lysins entsteht.

Ich muss ausdrücklich hervorheben, dass bisher keine Beweise für die Identität des aus Protaminen gewonnenen Lysins mit dem von Drechsel, Siegfried u. A. beschriebenen Lysin vorhanden sind. Einige Umstände deuteten sogar darauf hin, dass hier verschiedene Körper von der Zusammensetzung $\mathrm{C}_{6} \mathrm{H}_{14} \mathrm{~N}_{2} \mathrm{O}_{2}$ vorliegen, ebenso wie es verschiedene Leucine gibt.

Es möge noch erwähnt werden, dass bei dieser.Spaltung des Protamins keine Ammoniakbildung stattfindet.

\section{Die quantitativen Verhältnisse der Spaltungsprodukte.}

Zur Feststellung der Mengenverhältnisse, in denen die Protaminbasen bei der Spaltung der Protamine auftreten, wandte ich die eben beschriebene Trennungsmethode an.

Um das zeitraubende Trocknen zu umgehen, benutzte ich lufttrockene Präparate, deren Stickstoffgehalt jedesmal bei Beginn des Versuchs durch eine Bestimmung nach Kjeldahl ermittelt wurde. Von diesen Präparaten wurden dann 2,5-5,0 gr. mit Schwefelsäure gespalten und die Fällungen des Histidins 
und Arginins, letztere mit Hülfe von Silbersulfat, in der beschriebenen Weise gewonnen. Diese Niederschläge wurden mit Schwefelwasserstoff zerlegt, die Filtrate von den Schwefelmetallen nach sorgfältigem Auswaschen des Niederschlags eingedampft und eine genau gemessene Menge des Filtrats zur Stickstoffbestimmung nach Kjeldahl benutzt. In dieser Weise erhielt ich vier Stickstoffwerthe: 1. den Stickstoff des analysirten Protamins, 2. den des Histidins, 3. den des Arginins, 4. den des lysinhaltigen Filtrats.

In meiner früheren Mittheilung ${ }^{1}$ ) habe ich nachgewiesen, dass bei Weitem der grösste Theil der stickstoffhaltigen Spaltungsprodukte des Protamins durch Phosphorwolfram-. säure fällbar ist, dass aber, selbst wenn die Fällung in concentrirter Lösung vorgenommen wird, 6,4-6,7\% des gesammten Stickstoffs im Filtrat des Phosphorwolframsäureniederschlages nachzuweisen ist.

Ist diese Beobachtung etwa darauf zurückzuführen, dass die Protamine bei ihrer Spaltung Stoffe liefern, die durch Phosphorwolframsäure nicht gefällt werden, z. B. Monoamidosäuren? Sind also neben Lysin noch andere Stoffe, etwa Leucin oder ähnliche Amidosäuren, in dem Filtrat Nr. 4 vorhanden?

Ich habe bisher solche Stoffe nicht auffinden können und muss daher diese Frage verneinen. Auch ergibt sich eine genügende Erklärung für die eben angeführte Beobachtung aus der Löslichkeit der phosphorwolframsauren Salze von Histidin, Arginin und Lysin. Diese Löslichkeit ist so bedeutend, dass ich von der Phosphorwolframsäure als Fällungsmittel der Protaminbasen bei den quantitativen Bestimmungen völlig $\mathrm{Ab}$ stand genommen habe. Ich halte mich nach meinen Untersuchungen für berechtigt, den Stickstoff des Filtrats (Nr. 4) völlig auf Lysin zu beziehen.

Die bei den quantitativen Versuchen erhaltenen Resultate sind in folgenden Tabellen zusammengestellt.

1) Diese Zeitschrift, Bd. XXII, S. 176. 
Tabelle I.

\begin{tabular}{|c|c|c|c|c|c|c|c|c|c|}
\hline 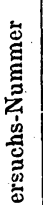 & $\begin{array}{c}\text { Bezeichnung } \\
\text { des } \\
\text { angewandten } \\
\text { Protamins }\end{array}$ & $\begin{array}{l}\text { Menge } \\
\text { des } \\
\text { Stick- } \\
\text { stoffs } \\
\text { der zer- } \\
\text { setzten } \\
\text { Sub- } \\
\text { stanz }\end{array}$ & \begin{tabular}{|} 
Stick- \\
stoff \\
des \\
Queck- \\
silber- \\
chlorid- \\
nieder- \\
schlages
\end{tabular} & $\begin{array}{c}\text { Stick- } \\
\text { stoff } \\
\text { des } \\
\text { Silber- } \\
\text { nieder- }\end{array}$ & \begin{tabular}{|c} 
Stick- \\
stoff \\
des \\
Filtrats
\end{tabular} & $\begin{array}{c}\text { Stick- } \\
\text { stoff } \\
\text { des } \\
\text { Queck- } \\
\text { silber- } \\
\text { chlorid- } \\
\text { nieder- } \\
\text { schlages }\end{array}$ & $\begin{array}{c}\text { Stick- } \\
\text { stoff } \\
\text { des } \\
\text { Silber- } \\
\text { nieder- } \\
\text { schlages }\end{array}$ & $\begin{array}{c}\text { Stick- } \\
\text { stoff } \\
\text { des } \\
\text { Filtrats }\end{array}$ & $\begin{array}{l}\text { Ver- } \\
\text { suchs- } \\
\text { fehler: } \\
\text { Stick- } \\
\text { stoff- } \\
\text { verlust }\end{array}$ \\
\hline & & in gr. & & & & \multicolumn{4}{|c|}{ in $\%$ des Gesammtstickstoffs } \\
\hline 1 & Clupein & 1,237 & 0,159 & - & - & 18,0 & - & - & - \\
\hline 2 & » & 0,7833 & 0,1281 & - & - & 16,3 & - & - & - \\
\hline 3 & » & 1,069 & 0,1848 & 0,7308 & 0,1176 & 17,3 & 68,3 & 11,0 & 3,4 \\
\hline 4 & Salmin & 0,5408 & 0,0875 & 0,3612 & 0,0735 & 16,1 & 66,8 & 13,6 & 3,5 \\
\hline 5 & Sturin & 0,9157 & 0,1575 & 0,5460 & 0,1624 & 17,2 & 59,6 & 17,7 & 5,5 \\
\hline 6 & 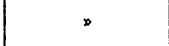 & 0,4240 & 0,0609 & 0,2688 & 0,0819 & 14,4 & 63,4 & 19,3 & 2,9 \\
\hline 7 & 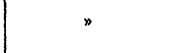 & 0,2875 & 0,0414 & 0,1652 & 0,0669 & 14,5 & 57,5 & 23,3 & 4,7 \\
\hline
\end{tabular}

Aus dieser Tabelle geht hervor, dass Clupein und Salmin bei der Spaltung annähernd die gleichen Mengen von Histidin, Arginin und Lysin liefern, dass hingegen das Sturin durchschnittlich weniger Histidin und Arginin und mehr Lysin ergibt.

Die bei der Spaltung des Clupeins und Salmins erhaltenen Stickstoffwerthe von Histidin, Arginin und Lysin stehen annähernd in dem Verhältniss 3:12:2, wie aus der folgenden Zusammenstellung zu ersehen ist.

Tabelle II.

\begin{tabular}{|c|c|c|c|c|c|}
\hline & Berechnet & \multicolumn{4}{|c|}{ Gefunden } \\
\hline & Stickstoffs & \multicolumn{3}{|c|}{ im Clupein } & \multirow{2}{*}{$\begin{array}{c}\mathrm{im} \\
\text { Salmin }\end{array}$} \\
\hline . & $\left|\begin{array}{r}\text { Verhältniss } \\
3 \mathrm{~N}: 12 \mathrm{~N}: 2 \mathrm{~N}\end{array}\right|$ & 1 & 2 & 3 & \\
\hline Histidin-Stickstoff & 17,6 & 18,0 & 16,3 & 17,3 & 16,1 \\
\hline Arginin-Stickstoff & 70,6 & - & 一 & 68,3 & 66,8 \\
\hline Lysin-Stickstoff & 11,8 & - & 一 & 11,0 & 13,6 \\
\hline Summe & 100 & - & 一 & 96,6 & $96, \tilde{0}$ \\
\hline
\end{tabular}


Zur Bestätigung dieser Resultate führte ich folgenden Versuch aus. Ich zerlegte eine nicht genauer bekannte Menge Clupeinsulfat mit Schwefelsäure und bestimmte in einer abgemessenen Menge der dem Volumen nach bekannten Reactionsflüssigkeit den Stickstoff mit Hülfe des Kjeldahl'schen Verfahrens. Hierdurch stellte ich die Menge des Gesammtstickstoffs fest.

Nachdem ich nun in einer anderen genau gemessenen Menge der Reactionsflüssigkeit die Schwefelsäure durch Baryt grösstentheils entfernt hatte, fällte ich Arginin und Histidin zusammen nach der oben beschriebenen Methode mit Hülfe von Silbersulfat und Baryt heraus und stellte in dem von Arginin und Histidin befreiten Filtrat die Stickstoffmenge fest. Dieselbe betrug $11,6 \%$ des Gesammtstickstoffs.

Auch diese Zahl stimmt mit den in obigen Tabellen für Lysin angeführten Werthen gut überein.

Man muss also aus diesen Untersuchungen den Schluss ziehen, dass auf 3 Atome Histidin-Stickstoff je 12 Atome Arginin-Stickstoff und 2 Atome Lysin-Stickstoff entstehen. Da nun das Histidin 3 Atome, das Arginin 4 Atome, das Lysin 2 Atome Stickstoff enthält, so ergibt sich, dass bei der Spaltung von Clupein und Salmin auf 1 Molekül Histidin je 3 Moleküle Arginin und je 1 Molekül Lysin entstehen müssen. Zählt man die in diesen Basen enthaltenen $\mathrm{C}$-, $\mathrm{H}-, \mathrm{N}-$, O-Atome zusammen, so gelangt man $\mathrm{zu}$ folgendem Resultat:

$$
\begin{aligned}
& 1 \text { Molekül Histidin } \mathrm{C}_{6} \mathrm{H}_{9} \mathrm{~N}_{4} \mathrm{O}_{2}=\mathrm{C}_{6} \mathrm{H}_{8} \mathrm{~N}_{4} \mathrm{O}_{2} \\
& 3 \gg \text { Arginin } \mathrm{C}_{6} \mathrm{H}_{14} \mathrm{~N}_{4} \mathrm{O}_{2}=\mathrm{C}_{18} \mathrm{H}_{48} \mathrm{~N}_{12} \mathrm{O}_{6} \\
& 1 \gg \text { Lysin } \mathrm{C}_{6} \mathrm{H}_{14} \mathrm{~N}_{2} \mathrm{O}_{2}=\frac{\mathrm{C}_{6} \mathrm{H}_{14} \mathrm{~N}_{2} \mathrm{O}_{2}}{\mathrm{C}_{30} \mathrm{H}_{65} \mathrm{~N}_{17} \mathrm{O}_{10}}
\end{aligned}
$$

Diese letztere Formel unterscheidet sich von der durch meine Analysen festgestellten Formel des Clupeins $\mathrm{C}_{30} \mathrm{H}_{57} \mathrm{~N}_{17} \mathrm{O}_{6}$ nur um $4 \mathrm{H}_{2} \mathrm{O}$. Man gelangt somit zu der folgenden einfachen Auffassung des Verhältnisses zwischen den genannten Protaminen und den Protaminbasen: Salmin und Clupein bilden sich, indem die 5 Moleküle der Protaminbasen in der Weise zusammentreten, dass je zwei benachbarte Moleküle ein Molekül Wasser verlieren. Dies entspricht folgender Zersetzungsgleichung: 


$$
\begin{aligned}
& \mathrm{C}_{30} \mathrm{H}_{57} \mathrm{~N}_{17} \mathrm{O}_{6}+4 \mathrm{H}_{2} \mathrm{O}=\mathrm{C}_{6} \mathrm{H}_{8} \mathrm{~N}_{8} \mathrm{O}_{2}+3 \mathrm{C}_{6} \mathrm{H}_{14} \mathrm{~N}_{4} \mathrm{O}_{2}+\mathrm{C}_{6} \mathrm{H}_{14} \mathrm{~N}_{2} \mathrm{O}_{2} \\
& \text { Salmin, Clupein Histidin Arginin Lysin. }
\end{aligned}
$$

Die bei der Spaltung des Sturins erhaltenen Zahlen zeigen besonders bezüglich des Lysins erhebliche Schwankungen, man kann sie annähernd auf das Verhältniss $3: 12: 4$ beziehen.

Die Zusammenstellung der gefundenen und der berechneten Werthe ergibt Folgendes.

\begin{tabular}{r|c|c|c|c}
\hline & $\begin{array}{c}\text { Berechnet in } \\
\text { Procenten des } \\
\text { Gesammt-Stick- } \\
\text { stoffs ffir das } \\
\text { verbältniss } \\
\text { 3. N: 12 N: 4N }\end{array}$ & \multicolumn{2}{|c|}{ Gefunden im Sturin } \\
\cline { 3 - 6 } & 1 & 2 & 3 \\
\hline Histidin-Stickstoff & 15,79 & 17,2 & 14,4 & 14,5 \\
\hline Arginin-Stickstoff & 63,16 & 59,6 & 63,4 & 57,5 \\
\hline Lysin-Stickstoff & 21,05 & 17,7 & 19,3 & 23,3 \\
\hline Summe & 100,00 & 94,5 & 97,1 & 95,3
\end{tabular}

Auch beim Sturin bestimmte ich den auf das Lysin fallenden Antheil des Gesammt-Stickstoffs direkt nach der auf voriger Seite angegebenen Methode und fand hierbei 18,3\% des Gesammt-Stickstoffs im Lysin.

Legt man das Verhältniss $3: 12: 4$ den Berechnungen zu Grunde, so kommt man zu dem Schluss, dass bei der Spaltung des Sturins ein Molekül Histidin neben drei Molekülen Arginin und zwei Molekülen Lysin entsteht. Addirt man die in den Formeln dieser Basen enthaltenen Atomzahlen, so erhält man als Summe: $\mathrm{C}_{36} \mathrm{H}_{79} \mathrm{~N}_{19} \mathrm{O}_{12}$.

Diese Formel unterscheidet sich von der oben aufgestellten Formel des Sturins $\mathrm{C}_{36} \mathrm{H}_{69} \mathrm{~N}_{19} \mathrm{O}_{7}$ um 5 Moleküle Wasser. Man kann sich also die Entstehung des Sturins ganz ähnlich vorstellen, wie die des Salmins und Clupeins. Hier vereinigen sich 6 Moleküle der Hexonbasen in der Weise, dass je zwei zusammentretende Moleküle ein Molekül Wasser verlieren. Die Gleichung für die Spaltung würde hiernach folgende sein:

$$
\underset{\text { Sturin }}{\mathrm{C}_{36} \mathrm{H}_{69} \mathrm{~N}_{19} \mathrm{O}_{7}}+5 \mathrm{H}_{2} \mathrm{O}=\underset{\text { Histidin }}{\mathrm{C}_{6} \mathrm{H}_{9} \mathrm{~N}_{8} \mathrm{O}_{8}}+\underset{\text { Arginin }}{3 \mathrm{C}_{6} \mathrm{H}_{14} \mathrm{~N}_{4} \mathrm{O}_{2}}+\underset{\text { Lysin. }}{\mathrm{C}_{6} \mathrm{H}_{14} \mathrm{~N}_{2} \mathrm{O}_{2}}
$$


Die Grösse des Moleküls der Protamine lässt sich aus den bisher vorliegenden Thatsachen ${ }^{1}$ ) nicht ersehen. Man muss also eigentlich für die Formel des Clupeins $\left(\mathrm{C}_{30} \mathrm{H}_{57} \mathrm{~N}_{17} \mathrm{O}_{6}\right) \times$ die des Sturins $\left(\mathrm{C}_{36} \mathrm{H}_{69} \mathrm{~N}_{19} \mathrm{O}_{7}\right) \times$ schreiben.

\section{Das Verhältniss der Protamine zu den Eiweisskörpern.}

Als die wichtigsten gemeinsamen Eigenschaften der Protamine und der Eiweisskörper im engeren Sinn betrachte ich folgende: 1. sie bilden Histidin, Arginin und Basen von der Zusammensetzung des Lysins; 2. sie geben die Biuretreaction; 3. sie werden durch Trypsin gespalten.

Minder wichtig sind: die Fällung mit Essigsäure und Ferrocyankalium, durch Pikrinsäure, Phosphorwolframsäure u. s. w., Bildung unlöslicher Verbindungen mit Benzoylchlorid und Linksdrehung der Ebene des polarisirten Lichtes.

Diejenigen Eigenschaften, welche nur einzelnen Gruppen von Eiweisskörpern zukommen, z. B. die Bildung aromatischer Produkte: Tyrosin (Millons Reaction), Amidophenylpropionsäure, Indol, Skatol, die Angreifbarkeit durch Pepsin, sind bei den Protaminen nicht vorhanden. Ausserdem vermisst man bei den Protaminen auch einige Eigenschaften, die bei den Eiweisskörpern weiter verbreitet sind: Schwefelgehalt, Bildung von Monoamidosäuren (Leucin u. s. w.) und die Rothfärbung mit Essigsäure und Schwefelsäure.

Bisher hat man bei physiologischen und chemischen Betrachtungen über das Eiweissmolekül die Aufmerksamkeit hauptsächlich auf die Monoamidosäuren als auf die charakteristischen Spaltungsprodukte gerichtet.

Wenn man nach meiner Anschauung die Protamine als die einfachsten Eiweisskörper betrachtet, so erscheint in den complicirteren Eiweisskörpern die basenbildende Gruppe als der eigentliche Kern des Eiweissmoleküls. Diese Anschauung setzt durchaus nicht voraus, dass die quantitativen Verhältnisse der Hexongruppen immer dieselben sind, wie sie sich in den bisher untersuchten Protaminen gezeigt haben. Es ist vielmehr

1) s. diese Zeitschrift, Bd. 22 S. 176. 
anzunehmen, dass der Kern der meisten Eiweisskörper bédeutend reicher an Lysingruppen ist, als das Salmin und Sturin.

In den einfachsten Eiweisskörpern, den Protaminen, liegt dieser Kern allein vor, die complicirteren Eiweissstoffe entstehen, indem an diesen Kern nach und nach andere Gruppen angefügt werden. Die Protamine bilden die erste Gruppe der Eiweisssubstanzen, die zweite Gruppe entsteht durch Anfügung von Monoamidosäuren der aliphatischen Reihe, z. B. von Leucin, Amidovaleriansäure, Asparaginsäure, Glutaminsäure, Glycocoll, an die basenbildende Gruppe.

In dies Molekül können andere Elemente eintreten. Fast immer erfolgt der Eintritt von Schwefel in mehr oder minder fest gebundenem Zustand, selten der Eintritt von Jod oder anderen Elementen. Zu diesen Körpern sind Leim und Spongin zu rechnen.

Andere Eiweisskörper liefern bei ihrer Spaltung ausser den Basen und den Monoamidosäuren der aliphatischen Reihe noch einen aromatischen Atomcomplex z. B. Tyrosin. Man könnte diese in zwei Hauptgruppen eintheilen, je nach dem sie Schwefel enthalten oder nicht.

Demgemäss würde zur dritten - schwefelfreien Gruppe das Fibroin der Seide zu rechnen sein, wohl auch die Peptone, sofern sich die Angabe mehrerer Autoren, dass sie schwefelfrei sind, bestätigen sollte.

Die vierte Gruppe endlich umfasst die grosse Zahl derjenigen Eiweissstoffe, welche neben den Basen, dem Leucin, Tyrosin und anderen Amidosäuren auch noch die schwefelhaltigen Zersetzungsprodukte ergeben.

Eine Mannigfaltigkeit wird dadurch hervorgerufen; dass die Angehörigen dieser einzelnen Gruppen sich mit einander verbinden können. Ich habe früher gezeigt, dass die Protamine sich mit Eiweisskörpern der vierten Gruppe vereinigen können, und habe es wahrscheinlich gemacht, dass auf diese Weise diejenigen Körper entstehen, die ich mit dem Namen Histone bezeichnet habe. Herr Dr. Kutscher hat neuerdings Verbindungen von Peptonen im älteren Sinne des Worts (nach 
Kühne's Bezeichnung: «Deuteroalbumosen») mit anderen Eiweisskörpern beschrieben.

Ein Theil der Eiweisskörper, die wir aus den Geweben der Thiere und Pflanzen extrahiren, stellt solche Verbindungen dar. Ein anderer Theil besteht aus Verbindungen der Eiweisskörper mit andersartigen prosthetischen Gruppen, die letzteren Produkte sind die «Proteide».

Es würde leicht sein, diese kurzen Andeutungen über die Eintheilung der Eiweissstoffe, welche sich aus meinen Anschauungen ergeben, zu vervollständigen oder zu modificiren. Ich hoffe, dass ich hierzu später. Gelegenheit finden werde.

Von allgemeiner Bedeutung ist die Analogie, welche nach meinen Untersuchungen zwischen den einfachsten Eiweisskörpern und den Kohlenhydraten besteht. Ebenso wie die höheren Polysaccharide durch Hydrolyse zunächst in Dextrine, weiterhin in Hexosen umgewandelt werden, so treten bei der Spaltung der hochmolekularen Protamine zunächst die Protone (Protamin-Peptone), weiterhin die Hexone auf. Die Zerlegung der höheren Polysaccharide in die einfacheren Kohlehydrate erfolgt mit Leichtigkeit durch die diastatischen Fermente. Meine in Gemeinschaft mit Herrn Mathews angestellten Untersuchungen bewiesen, dass es auch für die einfacheren Eiweissstoffe ein solches Ferment gibt, welches die Spaltung bis zu den Hexonen durchführt: das Trypsin. Dieser Zerfall erfolgt - ähnlich wie bei den Kohlehydraten - durch mehrere Zwischenstufen hindurch.

Meine Untersuchungen ergaben, dass die einfachsten Eiweisskörper, die Protamine, ebenso wie die Polysaccharide aus Gruppen, die je $\sigma$ Kohlenstoffatome enthalten, zusammengesetzt sind. Die Leichtigkeit, mit der die hydrolytische Spaltung bei den Polysacchariden erfolgt, hat zu der Schlussfolgerung geführt, dass die Bindung, welche hierbei gelöst wird, nicht etwa die gegenseitige Bindung zweier Kohlenstoffatome ist, sondern dass die einzelnen Monosaccharidgruppen durch Sauerstoffatome mit einander vereinigt werden und dass diese Vereinigung bei der Spaltung der Polysaccharide zerrissen wird. Dieselbe Schlussfolgerung ist auch für die Protamine zulässig, 
doch wird man hier auch die Frage erwägen müssen, ob die Vereinigung zwischen den Hexonmolekülen nicht vielleicht durch Imidgruppen gebildet wird.

Für die physiologischen Erörterungen über den Ursprung der Kohlehydrate aus eiweissartigen Stoffen oder ihre Verwandlung in solche ist es von grosser Bedeutung, festzustellen, ob die sechs Kohlenstoffatome innerhalb einer Hexongruppe direkt mit einander verbunden sind.

Nach Drechsel's Ansicht müsste dies bezüglich des Lysins der Fall sein, denn dieser Forscher hat bekanntlich das von ihm entdeckte Lysin als Diamidocapronsäure aufgefasst. E. Schulze hingegen, dessen wichtige Arbeitên über die Constitution des Arginins Licht zu verbreiten beginnen, ${ }^{1}$ ) neigt sich in Bezug auf diese letztere Base zu einer anderen Auffassung. Indess dürften die bisher vorliegenden Thatsachen, die Bildung von Harnstoff und Diamidovaleriansäure aus Arginin, noch kein Argument sein, welches die Annahme von sechs mit einander verbundenen Kohlenstoffatomen unwahrscheinlich macht. Besteht doch auch bei den Hexosen die Neigung, unter Bildung von Complexen mit 5 Atomen Kohlenstoff (Lävulinsäure) zu zerfallen. -

1) E. Schulze und E. Winterstein: Ueber ein Spaltungsprodukt des Arginins. Berichte der Deutschen chemischen Gesellschaft, Bd. 30 (1897), S. 2879. 